\title{
Detection of very-high-energy $\gamma$-ray emission from the colliding wind binary $\eta$ Car with H.E.S.S.
}

\begin{abstract}
H.E.S.S. Collaboration: H. Abdalla ${ }^{1}$, R. Adam ${ }^{26}$, F. Aharonian ${ }^{3,4,5}$, F. Ait Benkhali ${ }^{3}$, E. O. Angüner ${ }^{19}$, M. Arakawa ${ }^{37}$, C. Arcaro $^{1}$, C. Armand ${ }^{22}$, T. Armstrong ${ }^{41}$, H. Ashkar ${ }^{17}$, M. Backes ${ }^{8,1}$, V. Barbosa Martins ${ }^{33}$, M. Barnard ${ }^{1}$, Y. Becherini ${ }^{10}$, D. Berge ${ }^{33}$, K. Bernlöhr $^{3}$, R. Blackwell ${ }^{13}$, M. Böttcher ${ }^{1}$, C. Boisson ${ }^{14}$, J. Bolmont ${ }^{15}$, S. Bonnefoy ${ }^{33}$, J. Bregeon ${ }^{16}$, M. Breuhaus ${ }^{3}$, F. Brun ${ }^{17}$, P. Brun ${ }^{17}$, M. Bryan $^{9}$, M. Büchele ${ }^{32}$, T. Bulik ${ }^{18}$, T. Bylund ${ }^{10}$, S. Caroff ${ }^{15}$, A. Carosi $^{22}$, S. Casanova ${ }^{20,3}$, M. Cerruti ${ }^{15,42}$, T. Chand ${ }^{1}$, S. Chandra ${ }^{1}$, A. Chen $^{21}$, S. Colafrancesco ${ }^{21} \dagger$, G. Cotter ${ }^{41}$, M. Curyło ${ }^{18}$, I. D. Davids ${ }^{8}$, J. Davies ${ }^{41}$, C. Deil ${ }^{3}$, J. Devin ${ }^{24}$, P. deWilt ${ }^{13}$, L. Dirson ${ }^{2}$, A. Djannati-Ataï ${ }^{27}$, A. Dmytriiev ${ }^{14}$, A. Donath ${ }^{3}$, V. Doroshenko ${ }^{25}$, J. Dyks ${ }^{30}$, K. Egberts ${ }^{31}$, F. Eichhorn ${ }^{32}$, G. Emery ${ }^{15}$, J.-P. Ernenwein ${ }^{19}$, S. Eschbach ${ }^{32}$, K. Feijen ${ }^{13}$, S. Fegan ${ }^{26}$, A. Fiasson ${ }^{22}$, G. Fontaine ${ }^{26}$, S. Funk ${ }^{32}$, M. Füßling ${ }^{33}$, , S. Gabici ${ }^{27}$, Y. A. Gallant ${ }^{16}$, F. Gaté2 ${ }^{22}$, G. Giavitto ${ }^{33}$, L. Giunti ${ }^{27}$, D. Glawion ${ }^{23}$, J. F. Glicenstein ${ }^{17}$, D. Gottschall ${ }^{25}$, M.-H. Grondin ${ }^{24}$, J. Hahn ${ }^{3}$, M. Haupt ${ }^{33}$, G. Heinzelmann ${ }^{2}$, G. Henri ${ }^{28}$, G. Hermann $^{3}$, J. A. Hinton ${ }^{3}$, W. Hofmann ${ }^{3}$, C. Hoischen ${ }^{31}$, T. L. Holch ${ }^{7}$, M. Holler ${ }^{12}$, M. Hörbe ${ }^{41}$, D. Horns ${ }^{2}$, D. Huber ${ }^{12}$, H. Iwasaki ${ }^{37}$, M. Jamrozy $^{34}$, D. Jankowsky ${ }^{32}$, F. Jankowsky ${ }^{23}$, A. Jardin-Blicq ${ }^{3}$, V. Joshi ${ }^{32}$, I. Jung-Richardt ${ }^{32}$, M. A. Kastendieck ${ }^{2}$, K. Katarzyński ${ }^{35}$, M. Katsuragawa ${ }^{38}$,

U. Katz ${ }^{32}$, D. Khangulyan ${ }^{37}$, B. Khélifi ${ }^{27}$, J. King ${ }^{23}$, S. Klepser ${ }^{33}$, W. Kluźniak ${ }^{30}$, Nu. Komin ${ }^{21}$, K. Kosack ${ }^{17}$, D. Kostunin ${ }^{33}$, M. Kreter ${ }^{1}$,

G. Lamanna ${ }^{22}$, A. Lemière ${ }^{27}$, M. Lemoine-Goumard ${ }^{24}$, J.-P. Lenain ${ }^{15}$, E. Leser ${ }^{31,33, \star}$, C. Levy ${ }^{15}$, T. Lohse ${ }^{7}$, I. Lypova ${ }^{33}$, J. Mackey $^{4}$, J. Majumdar ${ }^{33}$, D. Malyshev ${ }^{25}$, D. Malyshev ${ }^{32}$, V. Marandon ${ }^{3}$, P. Marchegiani ${ }^{21}$, A. Marcowith ${ }^{16}$, A. Mares ${ }^{24}$, G. Martí-Devesa ${ }^{12}$, R. Marx $^{3}$, G. Maurin ${ }^{22}$, P. J. Meintjes ${ }^{36}$, R. Moderski ${ }^{30}$, M. Mohamed ${ }^{23}$, L. Mohrmann ${ }^{32}$, C. Moore ${ }^{29}$, P. Morris ${ }^{41}$, E. Moulin ${ }^{17}$, J. Muller ${ }^{26}$, T. Murach ${ }^{33}$, S. Nakashima ${ }^{40}$, K. Nakashima ${ }^{32}$, M. de Naurois ${ }^{26}$, H. Ndiyavala ${ }^{1}$, F. Niederwanger ${ }^{12}$, J. Niemiec ${ }^{20}$, L. Oakes ${ }^{7}$, P. O’Brien $^{29}$, H. Odaka ${ }^{39}$, S. Ohm ${ }^{33, \star}$, E. de Ona Wilhelmi ${ }^{33}$, M. Ostrowski ${ }^{34}$, M. Panter ${ }^{3}$, R. D. Parsons ${ }^{3}$, C. Perennes ${ }^{15}$, P.-O. Petrucci ${ }^{28}$, B. Peyaud ${ }^{17}$, Q. Piel $^{22}$, S. Pita ${ }^{27}$, V. Poireau ${ }^{22}$, A. Priyana Noel ${ }^{34}$, D. A. Prokhorov ${ }^{21}$, H. Prokoph ${ }^{33}$, G. Pühlhofer ${ }^{25}$, M. Punch ${ }^{27,10}$, A. Quirrenbach ${ }^{23}$, S. Raab ${ }^{32}$, R. Rauth $^{12}$, A. Reimer ${ }^{12}$, O. Reimer ${ }^{12}$, Q. Remy ${ }^{16}$, M. Renaud ${ }^{16}$, F. Rieger ${ }^{3}$, L. Rinchiuso ${ }^{17}$, C. Romoli ${ }^{3}$, G. Rowell ${ }^{13}$, B. Rudak ${ }^{30}$, E. Ruiz-Velasco ${ }^{3}$, V. Sahakian ${ }^{6}$, S. Sailer ${ }^{3}$, S. Saito ${ }^{37}$, D. A. Sanchez ${ }^{22}$, A. Santangelo ${ }^{25}$, M. Sasaki ${ }^{32}$, M. Scalici ${ }^{25}$, R. Schlickeiser ${ }^{11}$, F. Schüssler ${ }^{17}$, A. Schulz ${ }^{33}$,

H. M. Schutte ${ }^{1}$, U. Schwanke ${ }^{7}$, S. Schwemmer ${ }^{23}$, M. Seglar-Arroyo ${ }^{17}$, M. Senniappan ${ }^{10}$, A. S. Seyffert ${ }^{1}$, N. Shafi ${ }^{21}$, K. Shiningayamwe ${ }^{8}$, R. Simoni ${ }^{9}$, A. Sinha ${ }^{27}$, H. Sol ${ }^{14}$, A. Specovius ${ }^{32}$, S. Spencer ${ }^{41}$, M. Spir-Jacob ${ }^{27}$, Ł. Stawarz $^{34}$, R. Steenkamp ${ }^{8}$, C. Stegmann ${ }^{31,33}$, C. Steppa $^{31}$, T. Takahashi ${ }^{38}$, T. Tavernier ${ }^{17}$, A. M. Taylor ${ }^{33}$, R. Terrier ${ }^{27}$, D. Tiziani ${ }^{32}$, M. Tluczykont ${ }^{2}$, L. Tomankova $^{32}$, C. Trichard ${ }^{26}$, M. Tsirou $^{16}$, N. Tsuji ${ }^{37}$, R. Tuffs ${ }^{3}$, Y. Uchiyama ${ }^{37}$, D. J. van der Walt ${ }^{1}$, C. van Eldik ${ }^{32}$, C. van Rensburg ${ }^{1}$, B. van Soelen ${ }^{36}$, G. Vasileiadis ${ }^{16}$, J. Veh $^{32}$, C. Venter ${ }^{1}$, P. Vincent ${ }^{15}$, J. Vink ${ }^{9}$, H. J. Völk ${ }^{3}$, T. Vuillaume ${ }^{22}$, Z. Wadiasingh ${ }^{1}$, S. J. Wagner ${ }^{23}$, J. Watson ${ }^{41}$, F. Werner ${ }^{3}$, R. White ${ }^{3}$, A. Wierzcholska $^{20,23}$, R. Yang ${ }^{3}$, H. Yoneda ${ }^{38}$, M. Zacharias ${ }^{1}$, R. Zanin ${ }^{3}$, A. A. Zdziarski ${ }^{30}$, A. Zech ${ }^{14}$, J. Zorn $^{3}$, and N. Żywucka ${ }^{1}$
\end{abstract}

(Affiliations can be found after the references)

Received 23 September 2019 / Accepted 14 January 2020

\begin{abstract}
Aims. Colliding wind binary systems have long been suspected to be high-energy (HE; $100 \mathrm{MeV}<E<100 \mathrm{GeV}$ ) $\gamma$-ray emitters. $\eta$ Car is the most prominent member of this object class and is confirmed to emit phase-locked $\mathrm{HE} \gamma$ rays from hundreds of $\mathrm{MeV}$ to $\sim 100 \mathrm{GeV}$ energies. This work aims to search for and characterise the very-high-energy (VHE; $E>100 \mathrm{GeV}$ ) $\gamma$-ray emission from $\eta$ Car around the last periastron passage in 2014 with the ground-based High Energy Stereoscopic System (H.E.S.S.).

Methods. The region around $\eta$ Car was observed with H.E.S.S. between orbital phase $p=0.78-1.10$, with a closer sampling at $p \approx 0.95$ and $p \approx 1.10$ (assuming a period of 2023 days). Optimised hardware settings as well as adjustments to the data reduction, reconstruction, and signal selection were needed to suppress and take into account the strong, extended, and inhomogeneous night sky background (NSB) in the $\eta$ Car field of view. Tailored run-wise Monte-Carlo simulations (RWS) were required to accurately treat the additional noise from NSB photons in the instrument response functions.

Results. H.E.S.S. detected VHE $\gamma$-ray emission from the direction of $\eta$ Car shortly before and after the minimum in the X-ray lightcurve close to periastron. Using the point spread function provided by RWS, the reconstructed signal is point-like and the spectrum is best described by a power law. The overall flux and spectral index in VHE $\gamma$ rays agree within statistical and systematic errors before and after periastron. The $\gamma$-ray spectrum extends up to at least $\sim 400 \mathrm{GeV}$. This implies a maximum magnetic field in a leptonic scenario in the emission region of 0.5 Gauss. No indication for phase-locked flux variations is detected in the H.E.S.S. data.
\end{abstract}

Key words. astroparticle physics - radiation mechanisms: non-thermal - binaries: general - stars: individual: $\eta$ Car stars: Wolf-Rayet - cosmic rays

\footnotetext{
^ Corresponding authors: H.E.S.S. Collaboration, e-mail: contact.hess@hess-experiment.eu

$\dagger$ Deceased.
} 


\section{Introduction}

In 2009, the newly launched AGILE satellite and the Large Area Telescope (LAT) onboard the Fermi satellite detected a bright $\gamma$-ray source, which is coincident with the colliding wind binary (CWB) $\eta$ Car (Tavani et al. 2009; Abdo et al. 2009a). $\eta$ Car is composed of a luminous blue variable primary star of $\sim 100 M_{\odot}$ and an O- or B-type companion of $\sim 30 M_{\odot}$, and has been the object of numerous observations over centuries with ground-based telescopes as well as satellites, such as in radio, millimetre, infrared, optical, ultraviolet, and X-ray wavelengths (see e.g. Humphreys \& Martin 2012, for a review). The AGILE and Fermi-LAT detection, however, was the first time that highenergy $\gamma$-ray emission was seen from a CWB. The two member stars of $\eta$ Car orbit each other in a very eccentric orbit $(e \sim 0.9)$ with a period of $\sim 2023$ days (Damineli et al. 2008). Since the $\gamma$-ray detection in 2009, Fermi-LAT continued to monitor $\eta$ Car and has now covered almost two orbits (Balbo \& Walter 2017). The observed $\gamma$-ray emission is variable and composed of a lowand a high-energy component of which the latter extends up to $\sim 300 \mathrm{GeV}$ and shows orbit-to-orbit changes in the light-curve (Reitberger et al. 2012, 2015; Balbo \& Walter 2017).

A CWB system, such as $\eta$ Car, is typically composed of two massive stars that orbit each other and whose stellar winds form a colliding wind region (CWR) at the locations of ram-pressure balance. The CWR is characterised by a contact discontinuity and a strong shock on either side of it. The detection of nonthermal radio emission of prominent systems, such as WR 140, WR 146, and WR 147, have raised interest in this source class (see e.g. De Becker \& Raucq 2013, and references therein for a list of particle-accelerating CWBs). However, $\eta$ Car has not been identified in non-thermal radio emission yet (Duncan et al. 1995), which could be explained by a significant suppression due to the Razin effect (e.g. Falceta-Gonçalves \& Abraham 2012) or synchrotron self-absorption (e.g. Gupta \& Razzaque 2017). The first theoretical works studying particle acceleration in CWBs emerged in the 1980s and 1990s (e.g. Casse \& Paul 1980; Völk \& Forman 1982; Eichler \& Usov 1993; Romero et al. 1999) and were further extended in the following years (e.g. Mücke \& Pohl 2002; Benaglia \& Romero 2003; Reimer et al. 2006).

Both $\eta$ Car member stars drive strong and dense supersonic stellar winds. The primary star loses $\dot{M}_{1} \approx 2 \times 10^{-4} M_{\odot} \mathrm{yr}^{-1}$ in its $500 \mathrm{~km} \mathrm{~s}^{-1}$ fast wind (Pittard \& Corcoran 2002) ${ }^{1}$. The wind of the companion star has a mass-loss rate of $\dot{M}_{2} \approx 2 \times$ $10^{-5} M_{\odot} \mathrm{yr}^{-1}$, it is less dense than the primary wind, but is moving at considerably faster speeds of $v_{2} \approx 3000 \mathrm{~km} \mathrm{~s}^{-1}$ (Hillier et al. 2001; Pittard \& Corcoran 2002; Parkin et al. 2009). In the CWR, wind material is shock-heated to $50 \mathrm{MK}$ and gives rise to soft X-ray emission (Damineli 1996; Corcoran et al. 2015). Charged particles are predicted to be accelerated in the shocks on the primary and companion side of the contact discontinuity, reaching energies several orders of magnitude higher than those of the shock-heated plasma (see e.g. Reimer et al. 2006, and references therein).

The $\gamma$-ray spectrum as measured with Fermi-LAT exhibits two spectral components, one at low and one at high $\gamma$-ray energies. The lower-energy component shows a peak of the emission at $\sim 1 \mathrm{GeV}$, followed by a cutoff. A second, harder spectral component is visible in the $10-300 \mathrm{GeV}$ range (Farnier et al. 2011; Reitberger et al. 2012). Both components show variability at different levels along the orbit, with a prominent rise in the $10 \mathrm{GeV}-300 \mathrm{GeV}$ flux around periastron (Reitberger et al.

\footnotetext{
1 We note that Groh et al. (2012) find even higher primary mass-loss rates.
}

2015). The observed phase-locked flux variations on monthtimescales is nowadays seen as confirmation for the origin of the $\eta$ Car $\gamma$-ray emission in the binary system. The origin of the emission of the lower-energy component is discussed in the literature in different frameworks. While Farnier et al. (2011), Bednarek \& Pabich (2011), Balbo \& Walter (2017), Hamaguchi et al. (2018) favour a leptonic origin, Ohm et al. (2015) suggest a hadronic interpretation, where the emission stems from the decay of neutral pions that were produced in hadronic interactions. The high-energy component of the emission has been suggested to originate from hadrons (e.g. Farnier et al. 2011; Ohm et al. 2015; Reitberger et al. 2015; Balbo \& Walter 2017). In the CWR, electrons suffer strong inverse Compton and synchrotron losses, which makes acceleration to VHEs challenging and may limit the maximum energy electrons can be accelerated to. Above energies of $100 \mathrm{GeV}$ phase-locked flux variations due to absorption, modulated by orbital motion, are expected (e.g. Ohm et al. 2015). Measuring temporal variability of the emission is key to constrain the $\gamma$-ray emission region.

H.E.S.S. observed $\eta$ Car from 2004 to 2010 to search for VHE $\gamma$-ray emission from the binary and the Carina Nebula with the H.E.S.S. phase-I telescopes (Abramowski et al. 2012), but could only provide upper limits. Combined with the Fermi-LAT results, the H.E.S.S. non-detection of VHE $\gamma$-ray emission above $\sim 500 \mathrm{GeV}$ energies implies a cut-off of the accelerated particle population, or severe losses due to $\gamma-\gamma$ absorption in the strong stellar radiation fields. The original H.E.S.S. array was expanded by installing the CT5 telescope in 2012, which lowered the instrument's energy threshold significantly. This finally allows H.E.S.S. to reach into the domain of the $\eta$ Car $\gamma$-ray emission. Although detected in high-energy $\gamma$-rays over long exposures, one major limitation of Fermi-LAT is its comparably small detection area, which limits the sensitivity to study short-timescale changes in the $\eta$ Car light-curve. For instance, over a $\sim 6$-month period, the X-ray light-curve (Corcoran et al. 2017) shows a strong increase in flux shortly before periastron, followed by an X-ray flux minimum, which lasts for 30-60 days, and a recovery shortly thereafter. The highenergy $\gamma$-ray light-curves on the other hand only show significant detections in phase bins of typically 2.5 (5.0) months duration in the low (high) energy component (Reitberger et al. 2012). The energy range of Fermi-LAT at the highest energies and H.E.S.S. phase-II at the lowest energies cover the $\eta$ Car $\gamma$-ray spectrum in the cut-off region. H.E.S.S. compared to Fermi-LAT has a much larger collection area, which makes it very well suited to study short-timescale changes in the $\eta$ Car light-curve especially close to periastron. At $100 \mathrm{GeV}$ energies, the H.E.S.S. differential sensitivity of a $15-\mathrm{h}$ observation is a factor $\sim 50$ better than Fermi-LAT in a 30-day period (Hoischen et al. 2017), which motivates the H.E.S.S. observations presented here.

The paper is organised as follows: in Sect. 2 we discuss the H.E.S.S. instrument, the $\eta$ Car data set, and the data analysis. The impact of the strong and inhomogeneous night sky background (NSB) is also discussed. In Sect. 3 we conclude with placing the H.E.S.S. detection in the multiwavelength context and discuss it within the framework of particle acceleration and $\gamma$-ray production in $\eta$ Car.

\section{H.E.S.S. data analysis}

\subsection{H.E.S.S. data}

The H.E.S.S. experiment is an array of five Imaging Atmospheric Cherenkov Telescopes (IACTs) located in Namibia. It 
Table 1. Properties of the data sets used in this work to calculate the spectral points and the light-curve shown in Figs. 3 and 4, respectively.

\begin{tabular}{cccc}
\hline \hline $\begin{array}{c}\text { Data Set } \\
\text { (DS) }\end{array}$ & $\begin{array}{c}\text { Observation time } \\
\text { Modified Julian date (MJD) }\end{array}$ & Phase & $\begin{array}{c}\text { Live time } \\
\text { (h) }\end{array}$ \\
\hline I & $56439-56444$ & 0.78 & 0.9 \\
& $56666-56667$ & 0.89 & 0.8 \\
& $56741-56751$ & 0.93 & 9.4 \\
& $56775-56778$ & 0.95 & 0.9 \\
& $56797-56799$ & 0.96 & 1.8 \\
\hline II & $56803-56807$ & 0.96 & 3.3 \\
& $57066-57074$ & 1.09 & 8.6 \\
& $57077-57079$ & 1.10 & 4.0 \\
\hline All & $56439-57079$ & $0.78-1.10$ & 29.7 \\
\hline
\end{tabular}

Notes. The time interval of the H.E.S.S. observations, the total live time corresponding to the individual data sets, along with the covered orbital phase are summarised.

is the only IACT system integrating different telescope types into one array and is sensitive to $\gamma$-ray emission from Galactic and extragalactic sources in the energy range from $\sim 30 \mathrm{GeV}$ to tens of TeV. The four H.E.S.S.-I telescopes are equipped with mirrors of $12 \mathrm{~m}$ diameter and have a Davies-Cotton mirror geometry. The larger CT5 telescope has a parabolic mirror diameter of $28 \mathrm{~m}$, which makes it the largest IACT worldwide. The field of view (FoV) of the H.E.S.S.-I telescopes is $5^{\circ}$ in diameter, whereas CT5 has a FoV of $\sim 3.2^{\circ}$ (Bolmont et al. 2014). The addition of CT5 to the array significantly lowered the energy threshold, allowing for a broader overlap in energy in the transition region between H.E.S.S. and space-based instruments like Fermi-LAT.

The main challenge in the analysis of $\gamma$-ray data from the direction of $\eta$ Car is the large number of UV photons originating from the Carina Nebula that cause a significant increase in NSB, causing fake triggers of the telescope and increasing noise levels in the image pixels. For instance, the NSB rate in the Galactic plane is typically around $100 \mathrm{MHz}$ per pixel in the image. This translates to a mean number of NSB photons of 1.6 photoelectrons (p.e.) per pixel. The NSB in the direction of $\eta$ Car is up to ten times higher than the Galactic average and varies greatly across the FoV. To distinguish real Cherenkov showers from NSB-induced events, at least three neighbouring pixels with $>4.0$ p.e. above the average NSB are required to trigger the CT5 camera. The resulting telescope trigger rate is $1.5 \mathrm{kHz}$, mostly from genuine air showers. In the case of $\eta$ Car this threshold was applied for data set I (DS-I; see Table 1), while CT5 camera $\mathrm{HV}$ adjustments required to increase the pixel trigger thresholds to 4.5 p.e. for data set II (DS-II).

Observations in the direction of $\eta$ Car were mainly performed in the first six months of 2014 and 2015 (with the exception of one observation, which was conducted in May/June 2013). The properties of the individual data sets are summarised in Table 1. $\eta$ Car was observed with only CT5 for DS-II, which limits the analysis to monoscopic data. The data quality of DS-I and DS-II has been checked with standard tools. To adjust to the high NSB level and the extra pixels turned off to protect the Cherenkov camera, the requirement on the maximum relative fraction of deactivated pixels per observation in CT5 has been relaxed from 5 to $10 \%$ (cf. H.E.S.S. Collaboration 2017).

\subsection{H.E.S.S. data analysis}

The $\eta$ Car data have been analysed using two software packages with independent shower simulation, calibration, reconstruction and $\gamma$ /hadron separation to cross check the results. Results presented in this paper were produced with the model analysis (de Naurois \& Rolland 2009), which is based on a semianalytical model for the electromagnetic shower development in the atmosphere to infer the properties of the primary particle. All results have been successfully cross-checked in the $H A P$ framework, which employs a neural-network based shower reconstruction and $\gamma /$ hadron separation (Murach et al. 2015).

The NSB in the $\eta$ Car FoV as shown in Fig. 1 poses serious challenges for the instrument and the $\gamma$-ray data analysis. As described in Aharonian et al. (2004), the NSB rate can be inferred for every camera pixel without Cherenkov light signal based on the pedestal width. Additional checks and systematic tests have been performed to study its influence on the calibration, shower reconstruction, and $\gamma /$ hadron separation. These tests are described in more detail in the following.

The Carina Nebula is extended over $\mathrm{a} \gtrsim 0.3^{\circ}$ region, which is comparable to the Cherenkov image size of low-energy showers (cf. Fig. 1). Noise triggers could hence locally mimic low-energy extensive air showers. The goal was to reduce the impact of noise-induced triggers as early as possible in the analysis chain. Therefore, the main and cross-check analysis apply an image cleaning procedure to provide the seed for the likelihood fit (see below de Naurois \& Rolland 2009), and to reject noise pixels in the image analysis Aharonian et al. (2004), respectively. The nominal dual-threshold cleaning of 4 p.e. for a pixel and 7 p.e. for a neighbour pixel (i.e. $(4,7)$ cleaning) has been increased to a $(6,12)$ cleaning.

As a next step, a pixel-wise log-likelihood comparison between the predicted Cherenkov shower images, taking into account the NSB and electronic noise, and the images recorded by the telescopes is performed. This fit provides an estimate for the energy and direction of the incident particle (de Naurois \& Rolland 2009; H.E.S.S. Collaboration 2015). The semianalytical model also provides a goodness of fit parameter and the reconstructed primary interaction depth that are used to discriminate $\gamma$-ray events from hadronic background. In addition, the estimated error on the reconstructed direction of each event is required to be smaller than $0.3^{\circ}$.

To control the NSB, a uniform NSB goodness variable was defined, which characterises the likelihood of accidentally triggering on fluctuations caused by NSB. In contrast to the NSB goodness variable as introduced in H.E.S.S. Collaboration (2017), the uniform NSB goodness parameter assures a flat acceptance to background-like events, at the cost of an inhomogeneous acceptance to $\gamma$-ray like events. Although the number of accidental NSB camera triggers are reduced to a manageable level, NSB photons are still recorded, when real Cherenkovinduced events trigger the array and are recorded. The reduction in NSB-only events by applying these cuts and the response of the instrument is demonstrated in Fig. 1, right. Here, the acceptance to $\gamma$-ray like background events is shown for DS-I and DS-II. The asymmetry in RA and Dec expressed as $(x-(-x)) /(x+(-x))$ across the $\eta \mathrm{Car}$ FoV is stable at the $5-10 \%$ level. This shows that the influence of NSB on the background extraction is generally under control. For the generation of sky images, the ring background technique is used, while for the spectrum determination the reflected background method is used (Berge et al. 2007). 

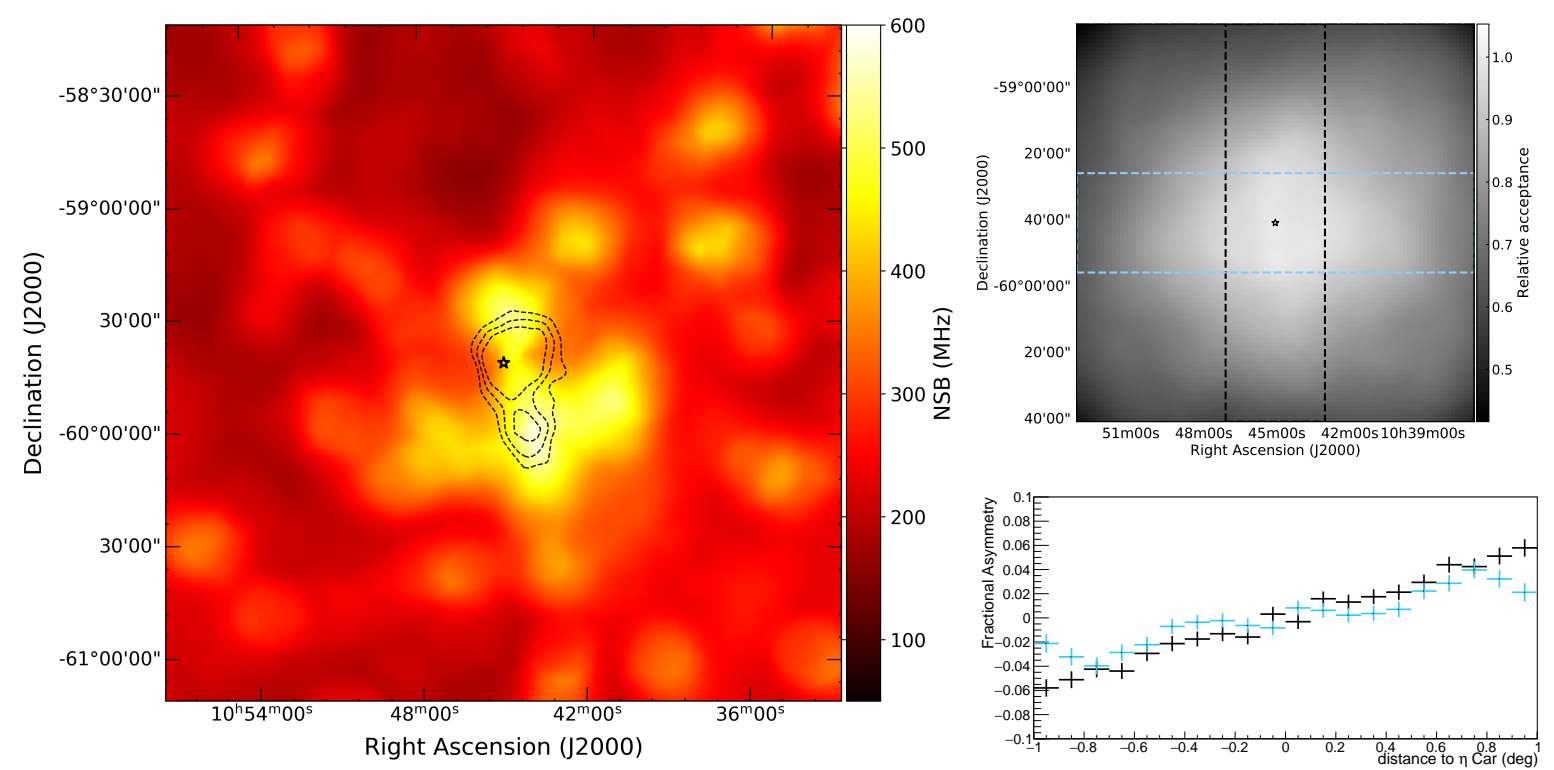

Fig. 1. Left: smoothed NSB rate per camera pixel in MHz for CT5 overlaid with contours for 3.5, 4.75 and $6 \sigma$ of the mono analysis of DS-I and DS-II. Turned-off pixels are treated as $0 \mathrm{MHz}$, hence the map illustrates the average NSB operating pixels are exposed to. The optical position of $\eta$ Car is denoted with a small star. Right: zoom into the 2D $\gamma$-ray acceptance map (top) and the relative asymmetry in RA and Dec (bottom).

\subsubsection{VHE $\gamma$-ray detection}

The analysis of data acquired in H.E.S.S. observations towards $\eta$ Car before 2011, and published in Abramowski et al. (2012), yielded an energy threshold of $470 \mathrm{GeV}$. For the CT5 mono analysis presented in this work, and the modifications made as described above, the energy threshold is 190 and $220 \mathrm{GeV}$ for DS-I and DS-II, respectively. Figure 2 shows the VHE $\gamma$-ray significance map of the sky in the direction of $\eta$ Car for all monoscopic events from both data sets that pass $\gamma$ /hadron separation cuts as described in the previous section. Significant VHE $\gamma$-ray emission is also seen in DS-I and DS-II separately. In the signal region, centred at the position of $\eta$ Car, a $\gamma$-ray excess of $(526 \pm 62)$ events above background at a significance level of $8.9 \sigma$ is found in DS-I. In DS-II, the $\gamma$-ray excess is $541 \pm 56$ at a statistical significance of $10.3 \sigma$. The emission seen in DS-I with the mono analysis is also confirmed, but at a lower significance of 7.2 $\sigma$, with the CT1-5 stereo analysis. This can partly be explained by the increased energy threshold of the stereo analysis. Also, the cross-check analysis confirms the significant detections in both data sets.

The maximum of the $\gamma$-ray emission shown in Fig. 2 stems from the direction of $\eta$ Car and not from the region of highest NSB. Outside of the signal region, the distribution of sky map significance is roughly consistent with the expected normal distribution, showing a width of 1.04 and 1.08 in DS-I and DS-II, respectively. The maximum of the NSB, as shown in Fig. 1, does however coincide with some residual $\gamma$-ray like emission south of $\eta$ Car. The origin of this feature and its implication for the $\eta$ Car measurement are discussed in the following.

As a first step, we attempt to determine the morphology of the $\gamma$-ray emission based on Fig. 2 and the $\gamma$-ray maps of the individual data sets. In ground-based $\gamma$-ray astronomy, instrument response functions (IRFs) are typically produced using Monte-Carlo (MC) simulations at fixed phase space points and assuming an ideal detector (no turned-off pixels, uniform NSB, no telescope tracking). The point spread function (PSF), however, is influenced by the high NSB level: NSB photons can enter the reconstruction, which leads to noisier shower images and

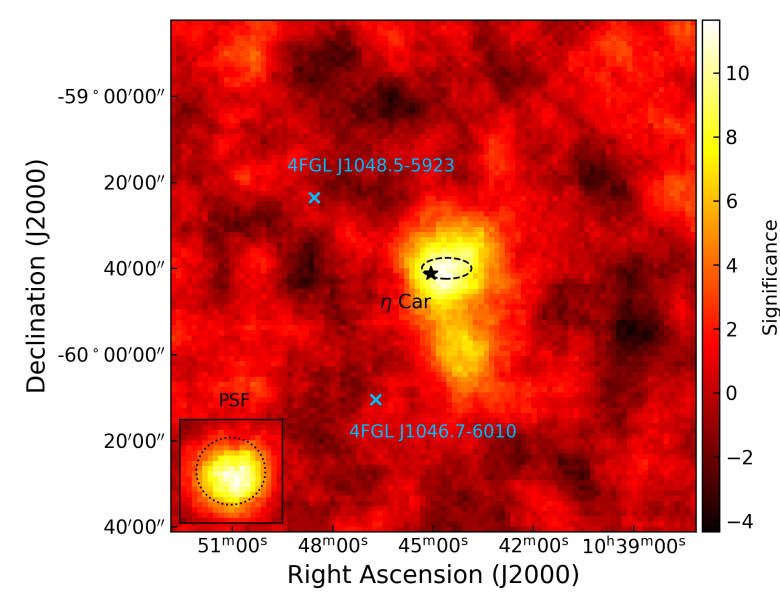

Fig. 2. Significance map as obtained in the CT5 mono analysis, for the combined DS-I $(0.78<p<0.96)$ and DS-II $(1.09<p<1.10)$ using an oversampling radius of $0.1^{\circ}$. The position of $\eta$ Car is denoted with a black star and the long-dashed ellipse refers to the uncertainty in the positional fit as described in the main text. The positions of the nearby Fermi-LAT sources 4FGL J1048.5-5923 and 4FGL J1046.7-6010 are shown with blue crosses (The Fermi-LAT Collaboration 2019). The inlay shows the point spread function (PSF) as obtained using run-wise simulations (RWS) in colour, while the overlaid dotted circle indicates the PSF using classical simulations.

hence an increased PSF. For complex FoVs and measurements at the systematic limit, this approach is not accurate enough anymore. It has been shown that a realistic description of the observing conditions and instrument properties under which $\gamma$ ray data has been taken is crucial when deriving the PSF of H.E.S.S. in measuring the extension of the Crab Nebula at TeV energies (H.E.S.S. Collaboration 2020). With its challenging observing conditions, the analysis presented here is another prime example for the utility of this approach. In the following, we employ the usage of run-wise simulations (RWS) to derive the PSF of DS-I and DS-II. Although this more realistic description represents a significant improvement over the classical IRFs, 
we note that due to computational limitations, only the $\gamma$-ray signal is simulated and not the cosmic-ray background, whose uniformity across the FoV is impacted by the increased and inhomogeneous NSB. A non-uniformity in cosmic-ray background impacts the precision of the background subtraction required to extract the $\gamma$-ray signal.

Positional fits of the $\gamma$-ray emission of DS-I and DS-II have been performed using RWS assuming different underlying spectral indices of the emission. Assuming a spectral index of $\Gamma=$ 3.7, consistent with the spectral result discussed below, the morphology fit on DS-II (the data set, which shows the lowest instrumental systematics) results in a morphology consistent with being point-like and a best-fit position of $\mathrm{RA}=10^{\mathrm{h}} 44^{\mathrm{m}} 35^{\mathrm{s}} \pm 6.6^{\mathrm{s}}$ and Dec $=-59^{\circ} 39^{\prime} 56.6^{\prime \prime} \pm 0.8^{\prime}(\mathrm{J} 2000), 3.8^{\prime}$ away from the optical $\eta$ Car position. To estimate the systematic uncertainty of the derived best-fit position of data taken under different observing and instrument conditions, morphology fits to DS-I and DSII in the RWS framework, and with classical IRFs in both the main and cross-check analysis were performed. The derived bestfit position depends, for instance, on the assumed underlying power-law spectral index, which was varied between $\Gamma=3.0$ and $\Gamma=4.0$. The systematic error on the position is estimated following this description as $\Delta \mathrm{RA}= \pm 1.5^{\mathrm{m}}$ and $\Delta \mathrm{Dec}= \pm 4.8^{\prime}$. Within the statistical and systematical error, the derived best-fit position is consistent with the optical position of $\eta$ Car. The consistent treatment of the NSB by using RWS is necessary to reconstruct a source that is compatible with being point-like at the $\eta$ Car position.

Another complication in the determination of the source morphology is the assumption of a single component as origin of the $\gamma$ rays. Figure 2 suggests that a weaker emission component south of $\eta$ Car exists and possibly biases the 2D morphology fit. A dedicated analysis was performed towards this emission component at $\mathrm{RA}=10^{\mathrm{h}} 44^{\mathrm{m}} 22.8^{\mathrm{s}}$ and $\mathrm{Dec}=-59^{\circ} 57^{\prime} 51.8^{\prime \prime}$ (J2000), which results in a hotspot at $6.5 \sigma$ level in DS-II, dubbed HOTS J1044-5957. At this significance level, and given the fact that the emission is located in the region with the highest NSB in the FoV, we do not claim a new source, but we note that not fully understood systematics could explain at least parts of this emission. The contribution of HOTS J1044-5957 to the $\eta$ Car emission is estimated to $\sim 15 \%$, based on a Gaussian fit to the 1D spatial profile along the axis connecting $\eta$ Car and HOTS J1044-5957.

We emphasise that the hotspot is not detected in the crosscheck analysis, neither in DS-I nor in DS-II. This further supports that the hotspot seen in the main analysis may be caused by a systematic effect, likely the response of the classifying parameters to the NSB. Also, at lower $\gamma$-ray energies, no counterpart is reported in the Fermi-LAT 4FGL catalogue (The Fermi-LAT Collaboration 2019). The spectral properties of $\eta$ Car as presented in the next section, are well compatible within errors in the main and cross-check analysis, implying a robust spectral measurement within uncertainties. To conclude, all tests confirm the integrity of the signal, and given the good positional coincidence, we assume in the following that it is connected to the CWB system. The potential impact of the systematics discussed above is addressed as systematic error on the inferred spectral measurement, as described in the next section.

\subsubsection{VHE $\gamma$-ray spectrum and light-curve}

In the following, the intrinsic $\gamma$-ray spectrum is reconstructed and the $\gamma$-ray signal is checked for variability and phase-locked flux variations as seen at other wavelengths. To enclose the entire

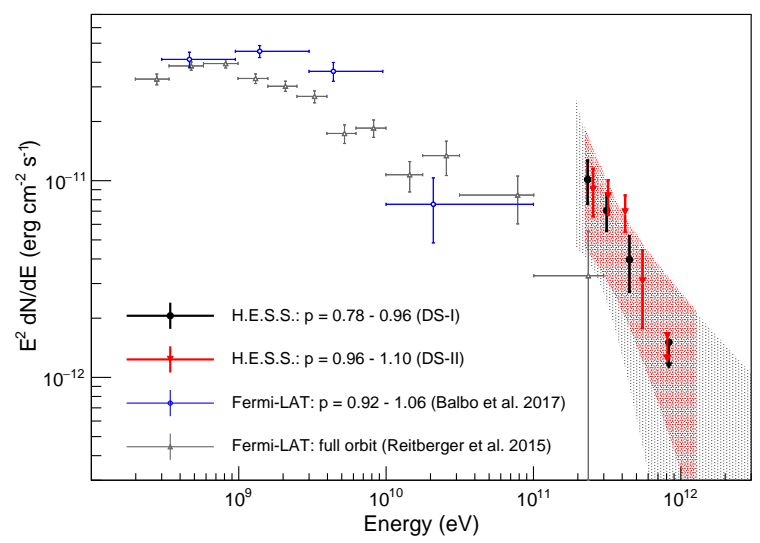

Fig. 3. Spectral energy distribution of $\eta$ Car for DS-I (black) and DS-II (red). H.E.S.S. points show $1 \sigma$ statistical errors. The shaded regions indicate the combined statistical and systematic errors (as given in the main text and Table 3). Fermi-LAT spectra from Reitberger et al. (2015) for the full orbit (grey) and for the last periastron passage from Balbo \& Walter (2017) (blue) are also shown.

emission seen from $\eta$ Car, the reflected background method (Berge et al. 2007) was used with an on-source region radius of $0.2^{\circ}$, and the forward-folding technique by Piron et al. (2001) is applied to extract spectral parameters. RWS are currently limited to the simulation of point sources of $\gamma$ rays. For the spectrum reconstruction one typically assumes that the response to $\gamma$-ray like background events is identical in the signal and background control regions. The number of turned-off pixels is much higher towards $\eta$ Car than in the surroundings. Hence, the response to $\gamma$-ray like background events is also different. For these reasons, we use in the following classical IRFs.

Figure 3 shows the spectra for DS-I and DS-II for the mono analyses. The best-fit spectral index for DS-I is $\Gamma_{\mathrm{DS}-\mathrm{I}}=$ $3.94 \pm 0.35_{\text {stat }}$ above an energy threshold of $190 \mathrm{GeV}$. The flux normalisation at the decorrelation energy ${ }^{2}$ of $E_{0, \mathrm{DS}-\mathrm{I}}=$ $290 \mathrm{GeV}$ is $F_{0, \mathrm{DS}-\mathrm{I}}=\left(5.1 \pm 0.5_{\text {stat }}\right) \times 10^{-11} \mathrm{ph} \mathrm{cm}^{-2} \mathrm{~s}^{-1} \mathrm{TeV}^{-1}$. The $\gamma$-ray spectrum for DS-II above an energy threshold of $220 \mathrm{GeV}$ is best described by a power-law with index of $\Gamma_{\mathrm{DS}-\mathrm{II}}=$ $3.49 \pm 0.23_{\text {stat }}$ and normalisation $F_{0, \text { DS-II }}=\left(3.2 \pm 0.3_{\text {stat }}\right) \times$ $10^{-11} \mathrm{ph} \mathrm{cm}^{-2} \mathrm{~s}^{-1} \mathrm{TeV}^{-1}$ at a decorrelation energy of $E_{0, \mathrm{DS}-\mathrm{II}}=$ $360 \mathrm{GeV}$. The DS-II spectrum is within statistical errors consistent with the DS-I spectrum (see Table 2).

Several sources of uncertainty influence these spectral measurements. They range from MC simulations of the air-shower and the instrument, the calibration, and data quality selection, to the high-level reconstruction, $\gamma$-ray selection, and background estimation. Table 3 lists all contributions to the flux normalisation and spectral index uncertainty and is based on H.E.S.S. Collaboration (2017). The systematic error from switched-off pixels has been increased from the nominal 5-10\% to reflect the conditions in this analysis. Errors on the spectral index and normalisation from uncertainties in the reconstruction and selection cuts are based on differences between the main and cross-check analysis. The downward systematic errors resulting from uncertainties in the background subtraction, as discussed at the end of the previous section, were estimated from the reconstructed integral flux of HOTS J1044-5957 in DS-II. Furthermore, we note that the reconstructed spectrum of the lead analysis may be prone to a shift of the energy scale of $20 \%$ towards lower energies. Even

\footnotetext{
2 The decorrelation energy $E_{0}$ is defined as $E_{0}=\exp \frac{\operatorname{cov}\left(F_{0}, \Gamma\right)}{F_{0} \Delta \Gamma^{2}} \mathrm{GeV}$ (Abdo et al. 2009b), where cov is the covariance error matrix.
} 
Table 2. Spectral statistics for the two analyses.

\begin{tabular}{cccccc}
\hline \hline DS & $\begin{array}{c}E_{\mathrm{th}} \\
{[\mathrm{TeV}]}\end{array}$ & $\Gamma$ & $\begin{array}{c}E_{0} \\
{[\mathrm{TeV}]}\end{array}$ & $\begin{array}{c}\Phi_{0} \\
{\left[\mathrm{~cm}^{-2} \mathrm{~s}^{-1} \mathrm{TeV}^{-1}\right]}\end{array}$ & $\begin{array}{c}\Phi(>0.2) \mathrm{TeV} \\
{\left[\mathrm{cm}^{-2} \mathrm{~s}^{-1}\right]}\end{array}$ \\
\hline I & 0.19 & $3.94 \pm 0.35$ & 0.29 & $(5.1 \pm 0.5) \times 10^{-11}$ & $(1.6 \pm 0.2) \times 10^{-11}$ \\
II & 0.22 & $3.49 \pm 0.23$ & 0.36 & $(3.2 \pm 0.3) \times 10^{-11}$ & $(2.0 \pm 0.2) \times 10^{-11}$ \\
\hline
\end{tabular}

Notes. Uncertainties are statistical. $E_{\mathrm{th}}$ denotes the threshold energy, $E_{0}$ the decorrelation energy, and $\Phi_{0}$ the flux at the decorrelation energy.

Table 3. Estimated contributions to the systematic uncertainties in the spectral measurements for the mono analyses presented in this work follow the description in H.E.S.S. Collaboration (2017) and we assume similar systematics as for the PG 1553+113 source, which has a similarly steep spectral index as $\eta$ Car.

\begin{tabular}{cccccc}
\hline \hline Source of uncertainty & Energy scale & $\begin{array}{c}\text { Flux } \\
\text { DS-I }\end{array}$ & $\begin{array}{c}\text { Index } \\
\text { DS-I }\end{array}$ & $\begin{array}{c}\text { Flux } \\
\text { DS-II }\end{array}$ & $\begin{array}{c}\text { Index } \\
\text { DS-II }\end{array}$ \\
\hline MC shower interactions & & $1 \%$ & & $1 \%$ & \\
MC atmosphere simulation & $7 \%$ & & & & \\
\hline $\begin{array}{c}\text { Instrument simulation/calibration } \\
\text { Broken pixels }\end{array}$ & $10 \%$ & $10 \%$ & & $10 \%$ & \\
Live time & & $10 \%$ & & $<5 \%$ & \\
\hline Reconstruction and selection cuts & $15 \%$ & $15 \%$ & 0.96 & $15 \%$ & 0.34 \\
Background subtraction & & $10 \% / 55 \%$ & 0.46 & $10 \% / 55 \%$ & 0.46 \\
\hline Total & $19 \%$ & $24 \% / 60 \%$ & 1.06 & $24 \% / 60 \%$ & 0.57 \\
\hline
\end{tabular}

Notes. Values quoted are for upward/downward or symmetric errors. See text for more details on the differences. The energy scale uncertainty in this analysis is estimated to be larger than for PG $1553+113$.

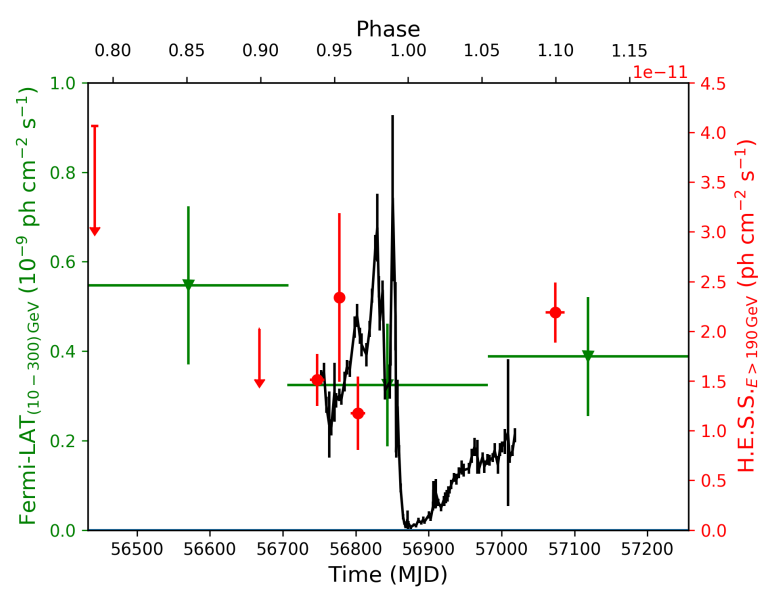

Fig. 4. Phase-binned H.E.S.S. flux above $190 \mathrm{GeV}$ as red points. The phase errors in the H.E.S.S. light-curve points refer to the re-grouped data given in Table 1. Fermi-LAT $\gamma$-ray flux points from Balbo \& Walter (2017) and Swift X-ray data from Corcoran et al. (2017) are shown as green triangles and as black line, respectively. Flux errors are all $1 \sigma$ while upper limits are $95 \%$ confidence level. The X-ray data has been scaled to arbitrary units for better comparison to the $\gamma$-ray data.

with this potential bias, the shifted spectral points would still be captured by the systematic error budget estimate presented in Table 3 and Fig. 3. We emphasise that the re-binned spectral points are shown only to guide the readers eye and cannot easily be combined with Fermi-LAT data points in a multiwavelength spectral fit.

Figure 4 shows the $\gamma$-ray light-curve of H.E.S.S. for the data described in Table 1. All flux points with a statistical significance of more than $2 \sigma$ are displayed as points, whereas upper limits at $95 \%$ confidence level are shown for non-significant flux measurements. The H.E.S.S. light-curve is within uncertainties consistent with no variability ${ }^{3}$. This is in agreement with the Fermi-LAT high-energy light-curve that is, however, rather limited in statistics and can only probe the $\gamma$-ray emission on much longer timescales. In the following, we put the H.E.S.S. results in the multiwavelength context and discuss what they imply for particle acceleration and $\gamma$-ray emission processes in this unique object.

\section{Discussion and outlook}

The H.E.S.S. measurement shows that there exists a VHE $\gamma$-ray source at a position coincident with the optical $\eta$ Car position. The emission is consistent with being point-like using RWS and the H.E.S.S. VHE $\gamma$-ray spectrum extends within statistical and systematic uncertainties at about $200 \mathrm{GeV}$ to the Fermi-LAT spectrum as presented in Balbo \& Walter (2017). The H.E.S.S. spectrum contains information about the maximum energy that the radiating particles in $\eta$ Car can reach. The last bin in the H.E.S.S. spectrum with a $>2 \sigma$ statistical significance starts at $\sim 400 \mathrm{GeV}$ in the two data sets and was confirmed by both analysis chains.

While the $100-10 \mathrm{GeV}$ emission detected by Fermi-LAT can be interpreted either in a leptonic or a hadronic scenario, there seems to be a preference in the community that the $\geq 10 \mathrm{GeV}$ emission can be best explained as originating from

3 The probability for a constant flux in nightly and monthly bins, including fits to the deep-exposure data sets at $p=0.95$ and $p=1.09$, is at the $0.5 \%$ level. When including systematic errors, this probability increases and the search for variability is non-significant at the $1.5-2.0 \sigma$ level. 
interacting protons (Farnier et al. 2011; Bednarek \& Pabich 2011; Ohm et al. 2015; Reitberger et al. 2015; Balbo \& Walter 2017). In the following, we elaborate on the implications of the maximum measured energy in the H.E.S.S. spectrum in a leptonic and hadronic scenario.

Assuming that particles are accelerated in the CWR via the diffusive shock acceleration process, the time to accelerate a charged particle to an energy $E_{\mathrm{TeV}}$ in a magnetic field $B_{\mathrm{G}}$ and in a shock with speed $v_{\mathrm{sh}, 10^{3} \mathrm{~km} \mathrm{~s}^{-1}}$ can be estimated as (Hinton \& Hofmann 2009):

$\tau_{\mathrm{acc}}=5 \times 10^{4} \eta v_{\mathrm{sh}, 10^{3} \mathrm{~km} \mathrm{~s}^{-1}}^{-2} \frac{E_{\mathrm{TeV}}}{B_{\mathrm{G}}} \mathrm{s}$,

where $B_{\mathrm{G}}$ is the magnetic field strength in Gauss, and $v_{\mathrm{sh}, 10^{3} \mathrm{~km} \mathrm{~s}^{-1}}$ is the shock speed in $10^{3} \mathrm{~km} \mathrm{~s}^{-1}$. The $\eta$ parameter is the diffusion coefficient expressed in terms of the Bohm diffusion coefficient, $\eta=\kappa / \kappa_{\mathrm{Bohm}} \geq 1$. In the most optimistic case, acceleration proceeds in the Bohm limit $(\eta=1)$, and with the shock speed set by the faster, companion star wind: $v_{\mathrm{w}}=v_{\mathrm{sh}}=3000 \mathrm{~km} \mathrm{~s}^{-1}$.

Assuming that the radiating particles are electrons, VHE $\gamma$ rays are produced by inverse Compton scattering off the photon fields from the two stars. Given their spectral temperature, inverse Compton scattering is proceeding into the Klein-Nishina regime, where most of the electron energy is transferred to the $\gamma$ ray, at tens of $\mathrm{GeV}$ energies. Hence, $\gamma$-ray emission up to $400 \mathrm{GeV}$ implies maximum electron energies in the same range. If adiabatic losses are neglected (cf. del Palacio et al. 2016), electrons at these energies predominantly suffer synchrotron and inverse Compton losses, while being accelerated. In the losslimited case, $\tau_{\text {acc }}=\tau_{\text {cool }}=\tau_{\text {IC }+ \text { sync }}$ applies. Assuming negligible inverse Compton losses, the cooling time can be approximated as:

$\tau_{\text {cool }}=4 \times 10^{2} E_{\mathrm{TeV}}^{-1} B_{\mathrm{G}}^{-2} \mathrm{~s}$.

For electrons to reach an energy of $400 \mathrm{GeV}$ in the synchrotron-loss limited acceleration case (where $\tau_{\text {acc }}=\tau_{\text {sync }}$ ), the maximum allowed magnetic field is estimated to be $\sim 0.5 \eta^{-1} \mathrm{G}$. With inverse Compton and adiabatic losses included, and a more realistic $\eta$ value, the maximum allowed magneticfield strength is reduced even further.

In a hadronic scenario, protons and heavier nuclei would have to be accelerated to at least $5 \mathrm{TeV}$ (e.g. Hinton \& Hofmann 2009). The maximum energy that protons can reach is to first order governed by the magnetic field strength and shock speed in the CWR for non-relativistic diffusive shock acceleration, and limited by the residence time of particles in the acceleration zone, as well as the particle density at the location of $p-p$ interaction. Typical densities in the CWR on the primary and companion side range between a few times $10^{9}$ and $\sim 10^{8}$ particles per $\mathrm{cm}^{3}$, respectively, which implies $p-p$ cooling times of the order of 5-100 days at phases considered here (e.g. Ohm et al. 2015). According to Eq. (1), and for magnetic field strengths of the order $\sim$ Gauss, proton energies $\geq 50 \mathrm{TeV}$ can be reached in the $p-p$ cooling-limited case and assuming Bohm diffusion. While the considerations made above might suggest a preference for a hadronic interpretation of the H.E.S.S. emission, the present data situation in the VHE domain does not allow to draw a firm conclusion.

The measurement of variability, or phase-locked flux variations on timescales of days to weeks, could help to identify the region of $\gamma$-ray production inside the CWR. H.E.S.S. observed $\eta$ Car shortly before the thermal X-ray maximum at phase $p \sim 0.95$ and after the X-ray minimum and recovery at phase $p \sim$ 1.1. The flux H.E.S.S. observed shows no indication of phase-locked flux variations. Due to the sporadic sampling and limited sensitivity of the measurement, no statement on variability on timescales shorter than months can be made. The lack of strong flux variations in the H.E.S.S. light-curve before and after the thermal X-ray minimum is broadly consistent with the behaviour in hard X-rays (Hamaguchi et al. 2018) and GeV $\gamma$ rays (e.g. Balbo \& Walter 2017). No flare similar to the one detected with AGILE (Tavani et al. 2009) in 2008 is seen in the H.E.S.S. data. Within statistical and systematic uncertainties, no change in the reconstructed source flux, nor a change in spectral index, could be detected. What seems apparent is that the steep spectrum observed with H.E.S.S. is in tension with models that assume negligible $\gamma \gamma$ absorption at $>100 \mathrm{GeV}$ energies (e.g Gupta \& Razzaque 2017). In fact, a strong phase-and energy-dependent suppression of the observed $\gamma$-ray emission around periastron passage is expected given the stellar parameters and the orbital configuration. Depending on the orbital solution (Madura et al. 2012), a $\tau_{\gamma \gamma}$ of 1-10 can be estimated, and leads to a strong suppression of the $\gamma$-ray flux in the H.E.S.S. energy range for the data presented here. Despite the comparably large systematics of the H.E.S.S. measurement in this data set, the sensitivity of the presented observations demonstrates the potential of ground-based $\gamma$-ray observations for the study of short-timescale flux changes in systems like $\eta$ Car (cf. Figs. 3 and 4).

The H.E.S.S. telescopes have been regularly monitoring $\eta$ Car since 2014. In this paper, we present data taken until 2015. The remaining data together with planned observations of the upcoming periastron passage in early 2020 will then conclude one full orbit of $\eta$ Car observations with H.E.S.S. and be covered in a future publication. They will allow us to test for temporal flux changes along the orbit. These observations also cover the phase of maximum stellar separation, where the high-energy $\gamma$-ray flux measured by Fermi-LAT reaches its minimum. More importantly, the H.E.S.S. observations of the upcoming periastron passage will be able to study the $\gamma$-ray emission on week-to-month timescales. This is crucial to probe the aforementioned X-ray maximum and minimum, which lasts between 30-60 days. The next-generation Cherenkov telescope array, CTA, will be able to improve considerably on the measured spectrum and light-curve. However, as demonstrated in this work, an accurate treatment of the increased NSB in the $\eta$ Car region and careful treatment of the data with tailored simulations are essential.

Acknowledgements. The support of the Namibian authorities and of the University of Namibia in facilitating the construction and operation of H.E.S.S. is gratefully acknowledged, as is the support by the German Ministry for Education and Research (BMBF), the Max Planck Society, the German Research Foundation (DFG), the Helmholtz Association, the Alexander von Humboldt Foundation, the French Ministry of Higher Education, Research and Innovation, the Centre National de la Recherche Scientifique (CNRS/IN2P3 and CNRS/INSU), the Commissariat à l'énergie atomique et aux énergies alternatives (CEA), the UK Science and Technology Facilities Council (STFC), the Knut and Alice Wallenberg Foundation, the National Science Centre, Poland grant no. 2016/22/M/ST9/00382, the South African Department of Science and Technology and National Research Foundation, the University of Namibia, the National Commission on Research, Science \& Technology of Namibia (NCRST), the Austrian Federal Ministry of Education, Science and Research and the Austrian Science Fund (FWF), the Australian Research Council (ARC), the Japan Society for the Promotion of Science and by the University of Amsterdam. We appreciate the excellent work of the technical support staff in Berlin, Zeuthen, Heidelberg, Palaiseau, Paris, Saclay, Tübingen and in Namibia in the construction and operation of the equipment. This work benefited from services provided by the H.E.S.S. Virtual Organisation, supported by the national resource providers of the EGI Federation. We thank Matteo Balbo, Klaus Reitberger as well as Mike Corcoran for providing us with multiwavelength data. 


\section{References}

Abdo, A. A., Ackermann, M., Ajello, M., et al. 2009a, ApJS, 183, 46 Abdo, A. A., Ackermann, M., Ajello, M., et al. 2009b, ApJ, 707, 1310 Abramowski, A., Acero, F., Aharonian, F., et al. 2012, MNRAS, 424, 128

Aharonian, F., Akhperjanian, A. G., Aye, K.-M., et al. 2004, Astropart. Phys., 22 109

Balbo, M., \& Walter, R. 2017, A\&A, 603, A111

Bednarek, W., \& Pabich, J. 2011, A\&A, 530, A49

Benaglia, P., \& Romero, G. E. 2003, A\&A, 399, 1121

Berge, D., Funk, S., \& Hinton, J. 2007, A\&A, 466, 1219

Bolmont, J., Corona, P., Gauron, P., et al. 2014, Nucl. Instrum. Methods Phys Res. A, 761, 46

Casse, M., \& Paul, J. A. 1980, ApJ, 237, 236

Corcoran, M. F., Hamaguchi, K., Liburd, J. K., et al. 2015, ArXiv e-prints [arXiv: 1507.07961]

Corcoran, M. F., Liburd, J., Morris, D., et al. 2017, ApJ, 838, 45

Damineli, A. 1996, ApJ, 460, L49

Damineli, A., Hillier, D. J., Corcoran, M. F., et al. 2008, MNRAS, 384, 1649

De Becker, M., \& Raucq, F. 2013, A\&A, 558, A28

de Naurois, M., \& Rolland, L. 2009, Astropart. Phys., 32, 231

del Palacio, S., Bosch-Ramon, V., Romero, G. E., \& Benaglia, P. 2016, A\&A 591, A139

Duncan, R. A., White, S. M., Lim, J., et al. 1995, Rev. Mex. Astron. Astrofis. Conf. Ser., 2, 23

Eichler, D., \& Usov, V. 1993, ApJ, 402, 271

Falceta-Gonçalves, D., \& Abraham, Z. 2012, MNRAS, 423, 1562

Farnier, C., Walter, R., \& Leyder, J. 2011, A\&A, 526, A57

Groh, J. H., Hillier, D. J., Madura, T. I., \& Weigelt, G. 2012, MNRAS, 423, 1623

Gupta, N., \& Razzaque, S. 2017, Phys. Rev. D, 96, 123017

Hamaguchi, K., Corcoran, M. F., Pittard, J. M., et al. 2018, Nat. Astron., 2, 731

H.E.S.S. Collaboration (Holler, M., et al.) 2015, in Proceedings of the 34th International Cosmic Ray Conference (ICRC2015), The Hague, The Netherlands

H.E.S.S. Collaboration (Abdalla, H., et al.) 2017, A\&A, 600, A89

H.E.S.S. Collaboration (Abdalla, H., et al.) 2020, Nat. Astron., 4, 167

Hillier, D. J., Davidson, K., Ishibashi, K., \& Gull, T. 2001, ApJ, 553, 837

Hinton, J. A., \& Hofmann, W. 2009, ARA\&A, 47, 523

Hoischen, C., Parsons, R. D., Garrigoux, T., Balzer, A., et al. 2017

Humphreys, R. M., \& Martin, J. C. 2012, Astrophys. Space Sci. Lib., 384, 1

Madura, T. I., Gull, T. R., Owocki, S. P., et al. 2012, MNRAS, 420, 2064

Mücke, A., \& Pohl, M. 2002, ASP Conf. Ser., 260, 355

Murach, T., Gajdus, M., \& Parsons, R. D. 2015, in Proceedings of the 34th International Cosmic Ray Conference (ICRC2015), The Hague, The Netherlands

Ohm, S., Zabalza, V., Hinton, J. A., \& Parkin, E. R. 2015, MNRAS, 449, L132

Parkin, E. R., Pittard, J. M., Corcoran, M. F., Hamaguchi, K., \& Stevens, I. R. 2009, MNRAS, 394, 1758

Piron, F., Djannati-Atai, A., Punch, M., et al. 2001, A\&A, 374, 895

Pittard, J. M., \& Corcoran, M. F. 2002, A\&A, 383, 636

Reimer, A., Pohl, M., \& Reimer, O. 2006, ApJ, 644, 1118

Reitberger, K., Reimer, O., Reimer, A., et al. 2012, A\&A, 544, A98

Reitberger, K., Reimer, A., Reimer, O., \& Takahashi, H. 2015, A\&A, 577, A100

Romero, G. E., Benaglia, P., \& Torres, D. F. 1999, A\&A, 348, 868

Tavani, M., Sabatini, S., Pian, E., et al. 2009, ApJ, 698, L142

The Fermi-LAT collaboration 2019, ApJS accepted, [arXiv:1902 . 10045]

Völk, H. J., \& Forman, M. 1982, ApJ, 253, 188

${ }^{1}$ Centre for Space Research, North-West University, Potchefstroom 2520, South Africa

2 Institut für Experimentalphysik, Universität Hamburg, Luruper Chaussee 149, 22761 Hamburg, Germany

3 Max-Planck-Institut für Kernphysik, PO Box 103980, 69029 Heidelberg, Germany

${ }^{4}$ Dublin Institute for Advanced Studies, 31 Fitzwilliam Place, Dublin 2, Ireland

5 High Energy Astrophysics Laboratory, RAU, 123 Hovsep Emin St Yerevan 0051, Armenia

6 Yerevan Physics Institute, 2 Alikhanian Brothers St., 375036 Yerevan, Armenia

7 Institut für Physik, Humboldt-Universität zu Berlin, Newtonstr. 15, 12489 Berlin, Germany

${ }^{8}$ Department of Physics, University of Namibia, Private Bag 13301, Windhoek, Namibia

9 GRAPPA, Anton Pannekoek Institute for Astronomy, University of Amsterdam, Science Park 904, 1098 XH Amsterdam, The Netherlands
10 Department of Physics and Electrical Engineering, Linnaeus University, 35195 Växjö, Sweden

11 Institut für Theoretische Physik, Lehrstuhl IV: Weltraum und Astrophysik, Ruhr-Universität Bochum, 44780 Bochum, Germany

12 Institut für Astro- und Teilchenphysik, Leopold-FranzensUniversität Innsbruck, 6020 Innsbruck, Austria

${ }^{13}$ School of Physical Sciences, University of Adelaide, Adelaide 5005, Australia

14 LUTH, Observatoire de Paris, PSL Research University, CNRS, Université Paris Diderot, 5 place Jules Janssen, 92190 Meudon, France

15 Sorbonne Université, Université Paris Diderot, Sorbonne Paris Cité, CNRS/IN2P3, Laboratoire de Physique Nucléaire et de Hautes Energies, LPNHE, 4 place Jussieu, 75252 Paris, France

${ }^{16}$ Laboratoire Univers et Particules de Montpellier, Université Montpellier, CNRS/IN2P3, CC 72, Place Eugène Bataillon, 34095 Montpellier Cedex 5, France

17 IRFU, CEA, Université Paris-Saclay, 91191 Gif-sur-Yvette, France

18 Astronomical Observatory, The University of Warsaw, Al. Ujazdowskie 4, 00-478 Warsaw, Poland

19 Aix-Marseille Université, CNRS/IN2P3, CPPM, Marseille, France

${ }^{20}$ Instytut Fizyki Jạdrowej PAN, ul. Radzikowskiego 152, 31-342 Kraków, Poland

${ }^{21}$ School of Physics, University of the Witwatersrand, 1 Jan Smuts Avenue, Braamfontein, Johannesburg 2050, South Africa

${ }^{22}$ Laboratoire d'Annecy de Physique des Particules, Univ. Grenoble Alpes, Univ. Savoie Mont Blanc, CNRS, LAPP, 74000 Annecy, France

23 Landessternwarte, Universität Heidelberg, Königstuhl, 69117 Heidelberg, Germany

${ }^{24}$ Université Bordeaux, CNRS/IN2P3, Centre d'Études Nucléaires de Bordeaux Gradignan, 33175 Gradignan, France

${ }^{25}$ Institut für Astronomie und Astrophysik, Universität Tübingen, Sand 1, 72076 Tübingen, Germany

${ }^{26}$ Laboratoire Leprince-Ringuet, École Polytechnique, CNRS, Institut Polytechnique de Paris, 91128 Palaiseau, France

27 APC, AstroParticule et Cosmologie, Université Paris Diderot, CNRS/IN2P3, CEA/Irfu, Observatoire de Paris, Sorbonne Paris Cité, 10 rue Alice Domon et Léonie Duquet, 75205 Paris Cedex 13, France

28 Univ. Grenoble Alpes, CNRS, IPAG, 38000 Grenoble, France

29 Department of Physics and Astronomy, The University of Leicester, University Road, Leicester, LE1 7RH, UK

${ }^{30}$ Nicolaus Copernicus Astronomical Center, Polish Academy of Sciences, ul. Bartycka 18, 00-716 Warsaw, Poland

${ }^{31}$ Institut für Physik und Astronomie, Universität Potsdam, KarlLiebknecht-Strasse 24/25, 14476 Potsdam, Germany

${ }^{32}$ Friedrich-Alexander-Universität Erlangen-Nürnberg, Erlangen Centre for Astroparticle Physics, Erwin-Rommel-Str. 1, 91058 Erlangen, Germany

${ }^{33}$ DESY, 15738 Zeuthen, Germany

${ }^{34}$ Obserwatorium Astronomiczne, Uniwersytet Jagielloński, ul. Orla 171, 30-244 Kraków, Poland

35 Centre for Astronomy, Faculty of Physics, Astronomy and Informatics, Nicolaus Copernicus University, Grudziadzka 5, 87-100 Torun, Poland

36 Department of Physics, University of the Free State, PO Box 339, Bloemfontein 9300, South Africa

37 Department of Physics, Rikkyo University, 3-34-1 Nishi-Ikebukuro, Toshima-ku, Tokyo 171-8501, Japan

${ }^{38}$ Kavli Institute for the Physics and Mathematics of the Universe (WPI), The University of Tokyo Institutes for Advanced Study (UTIAS), The University of Tokyo, 5-1-5 Kashiwa-no-Ha, Kashiwa, Chiba, 277-8583, Japan

39 Department of Physics, The University of Tokyo, 7-3-1 Hongo, Bunkyo-ku, Tokyo 113-0033, Japan

${ }^{40}$ RIKEN, 2-1 Hirosawa, Wako, Saitama 351-0198, Japan

41 Department of Physics, University of Oxford, Denys Wilkinson Building, Keble Road, Oxford OX1 3RH, UK

42 Now at Institut de Ciències del Cosmos (ICC UB), Universitat de Barcelona (IEEC-UB), Martí Franquès 1, 08028 Barcelona, Spain 